\title{
A Review of Guided Filter Based Pansharpening Methods
}

\author{
Yanan Gao, Yiming Zhang,Xu Li,Lixin Li, Ang Gao,YuPan \\ School of Electronics and Information, Northwestern Polytechnical University, China \\ E-mail:gaoyanan@mail.nwpu.edu.cn
}

\begin{abstract}
Pansharpening aims at injecting the spatial structure of the high resolution panchromatic(PAN) image into the low resolution multispectral(MS) image. In order to overcome the shortcomings of the existing methods that can not extract the details of the image accurately, some pansharpening methods based on guided filter have been proposedinrecent two years. This kind of filter has a good edge-preserving performance, which has been widely used in detail enhancement, compression and many other image processing fields. In this paper, we try to review several guided filtering based pansharpening methods includingMGF, GFIHS, GFCS-B/GFCS-M, and Meng's method.We also implement some other methods without guided filter.The advantages and disadvantages of all methodsare analyzed in detail.Experiments carried out on IKONOS and QuickBird satellite images confirm that guide filter for fusion of remote sensing images has a potential prospect.

Keywords: pansharpening; guided filter; panchromatic; multispectral
\end{abstract}

\section{Introduction}

Remote sensing images as an important source of information are used to describe the surface on earth. But due to the technical limitations of the sensors and other factors, many existing observation satellites such as IKONOS, QuickBird, GeoEye-1, and WorldView-2 can't provide ideal images directly.They yield high spatial resolution panchromatic(PAN) image and lowspatial resolution multispectral(MS) image instead. Pansharpening is a process of merging MS and PAN images to enhance the spatial resolution of MS image. The fused image has both greatspatial and spectral resolutions[1].

A large number of pansharpening methodshave been proposed over the past two decades.Some well-known classical methods like Principal Component Analysis(PCA)[2], Gram Schmidt(GS)[3], Discrete Wavelet Transform(DWT)[4], and Generalized Intensity-Hue-Saturation(GIHS)[5] have played a decisive boost for the development of remote sensing image fusion. But these methods often leadto the problems of spectral distortion and partialspatial information loss. Then Leung et al.[6]developed an improved adaptive Intensity-Hue-Saturation(IAIHS)method, who designed a moreadaptive weighting matrix in the spatial details injection step, however, the spectral distortion of the fused image also has a deviationboth in visual appearance and spectral values.In recent years, aiming at improving the quality of the fused image, several pansharpening methodsbegan to use edge-preserving image filters. Guided filter is a new and effective edge-preserving smoothing filter which was first proposed by He Kaiminget al.in 2011 [7]. It is derived from a local linear model and has better behavior near edges than other edge-preserving filters. In addition, it has a fast and non-approximate linear time algorithm. Hence, the guided filter appears in several recent proposed pansharpening methods.In 2015, Kishor P. Uplaet al.[8] proposed a multistage guided filter(MGF)which aims to extract the details from both MS and PAN images, but it's difficult to determine the guided filter decomposition levels and has a strong reliance on data.Amina Jameel et al. [9]combined the guided filter and IHS method(GFIHS) in 2016, andthe result has high spectral fidelity but with low spatial resolution.Liu Junminet al.[10] proposed two novel guided filter based pansharpening methods(GFCS-B and GFCS-M) in 2016 to reduce the redundant details among the MS and PAN images, but deciding which isbetter between GFCS-B and GFCS-M relies more on the selected image. In the same year, a three-layer-decomposition fusion method with guided filter was proposed by Meng Xiangchaoet al.[11]. In this method, the PAN image is decomposed into three layers: a strong edge layer, a detail layer, and a low-frequency layer. The method is also strongly dependent on the data. The result from IKONOS satellite images is not satisfied. In this paper, we implement these methods based on guided filter, such asMGF, GFIHS, GFCS-B/GFCS-M,and Meng's method, as well as others which do not use this filter, such as GS, GIHS, and IAIHS. Experiments are carried out on IKONOS and QuickBird satellite images. The subjective and objective analyses confirm that guided filter based pansharpening methods have the spatial details more similar to the PAN image and spectral information very close to MS image.

The rest of this paper is organized as follows. Section 2 briefly reviews the guided image filtering algorithm. Then,four types of fusion algorithms based on guided filter are described in Section 3.The experimental results and discussions are presented in Section 4. Finally, Section 5 concludes the paper. 


\section{Guided image filtering[7]}

The guided filter is an effective edge-preserving smoothing filter that is based on a local linear model. It has been successfully applied in many fields because of its good properties, such as edge-awaresmoothing, detail enhancement, image matting, imagefeathering, dehazing and so on. The guided filter involves an input image $\boldsymbol{P}$, a guidance image $\boldsymbol{G}$, and an output image $\boldsymbol{Q}$. The output image $\boldsymbol{Q}$ is assumed to be a linear transformation of theguidance image $G$ in a local window $w_{k}$ centered at pixel $k$ :

$$
\boldsymbol{Q}_{i}=a_{k} \boldsymbol{G}_{i}+b_{k}, \forall i \in w_{k}(1)
$$

where $\boldsymbol{G}_{i}$ and $\boldsymbol{Q}_{i}$ denote the $i$ th pixel of the guidance and output images in window $w_{k}, w_{k}$ is a square window of size $(2 r+1) \times(2 r+1)$.ris the radius of the window. $a_{k}$ and $b_{k}$ are linear coefficients assumed to be constant in $w_{k}$ and can be determined by minimizingthe squared difference between the output $\boldsymbol{Q}$ and the input $\boldsymbol{P}$.

$$
E\left(a_{k}, b_{k}\right)=\sum_{i \in w_{k}}\left(\left(a_{k} \boldsymbol{G}_{i}+b_{k}-\boldsymbol{P}_{i}\right)^{2}+\varepsilon a_{k}^{2}\right)(2)
$$

where $\mathcal{E}$ is a regularization parameter. The coefficients $a_{k}$ and $b_{k}$ can be determined by the following:

$$
\begin{aligned}
& a_{k}=\frac{\frac{1}{|w|} \sum_{i \in w_{k}}\left(\boldsymbol{G}_{i} \boldsymbol{P}_{i}-\mu_{k} \bar{p}_{k}\right)}{\sigma_{k}^{2}+\varepsilon} \\
& b_{k}=\bar{p}_{k}-a_{k} \mu_{k}(4)
\end{aligned}
$$

Here, $\mu_{k}$ and $\sigma_{k}^{2}$ are the mean and variance of $\boldsymbol{G}$ in $w_{k}$ respectively, $|w|$ is the number of all the pixels in $w_{k}$, and $\bar{p}_{k}$ is the mean of $\boldsymbol{P}$ in $w_{k}$. As we can see, the $i$ th pixel has different values in all the overlapping windows $w_{k}$ that cover $i$. In order to overcome the problem, it uses a strategy that averages all the possible values of $a_{k}$ and $b_{k}$, then the guided filter is estimated as the following:

$$
\boldsymbol{Q}_{i}=\bar{a}_{i} \boldsymbol{G}_{i}+\bar{b}_{i}(5)
$$

where $\bar{a}_{i}=\frac{1}{|w|} \sum_{k \in w_{i}} a_{k}, \bar{b}_{i}=\frac{1}{|w|} \sum_{k \in w_{i}} b_{k}$.

\section{Pansharpening methods based on guided filter}

Recently, guided filter is employed in image fusion by researchers.Li etal. [12]firstly extended the guided filter for fusion of multifocus, multitemporal, multiexposure and medical images in 2013. Then MGF method [8] applied guide filter to remote sensing image fusion in 2015 for the first time. In this section, several pansharpening methods for multispectral(MS) and panchromatic(PAN) images based on guided filter are introduced.

\subsection{MGF method[8]}

In this method, the authors proposed a multiresolution image fusion approach based on multistage guided filter(MGF). In order to extract more meaningful details from both MS as well as PAN image, they extended the guided filter to a multistage form. The detail extraction process exploited the relationship between the PAN and MS images by utilizing one of them as a guidance image while extracting details from the other. In this way, the spatial distortion of the MS image is reduced by consistently combining the details obtained using both types of images, so the final fused image has better preservation of spatial and spectral details. The authorsdescribed a concrete multistage decomposition method in the paper.

MGF pansharpening method can be described as follows:

(1) MS image is resampled to the size of the PAN image by using a suitable interpolation technique(bicubic interpolation is normally preferred).

(2) Compute the intensity component that is obtained using a weighted average of the four MS bands.

(3) The intensity component of the MS image and the PAN image are used for multistage guided filter.Then, detail and approximation layers are obtained. The authors used two-stage guided filter decompositions.

(4) Calculate the weighted gain separately for each pixel of the four MS bands. The final fused image is obtained by adding sum of the extracted high frequency details with the gain to the corresponding MS image. 


\subsection{GFIHS method[9]}

To overcome the spectral distortion, the authors proposed an improved guided filter and IHS technique. The scheme combines the high resolution panchromatic and low resolution multispectral images taking into account the intensity levels and spatial information. IHS is used for image decomposition and the guided filter is used to further refine the weight matrix for each pixel. The use of weight matrix in the final step not only increases the spectral quality but also improves the spatial quality of the fused image.

GFIHS pansharpening method can be described as follows:

(1) MS image is resampled to the size of the PAN image by using bicubic interpolation. Compute the intensity component obtained by least square regression.

(2) Let the PAN image pass through a Laplacian filter of size $3 \times 3$ to obtain the high-pass filtered image.

(3) Construct the initial weight. Then, the initial weight and high-pass filtered image pass through a guided filter to obtain the refined weights for spatial detail injection.

(4) In order to simultaneously improve both the spatial and the spectral quality, one weight matrix is selected for all the bands. Calculate the average gray level value of each band. Then the weight matrix corresponding to the multispectral band having the highest mean value is then used for spatial detail injection step.The detail is obtained by subtracting intensity component from PAN image. Adding the detail with the weight matrix to MS image to get the final fused image.

\subsection{GFCS-B/GFCS-M method[10]}

Based on the guided filter, the authors proposed two novel pansharpening methods in order to reduce the redundant details among the MS and PAN images. With the help of the MS image, the authors extracted the missing spatial details of the MS image by minimizing the difference between the PAN image and its corresponding filtered output. The two proposed methods were referred to as guided filter based component substitution with a band-dependent version(GFCS-B) and a multispectral version(GFCS-M) pansharpening methods.

The main difference between the GFCS-B method and GFCS-M method is the manner of the guidance image. GFCS-B method applies the guided filter to the histogram matched PAN image with each MS band as the guidance, while GFCS-M method is based on the multi-channel version of the guided filter with all MS bands as the guidance images.

This method can be described as follows:

(1) The MS bands are interpolated into the same scale of the PAN image by using bicubic interpolation.

(2) Perform the histogram matching between the PAN image and the intensity component(or each band of MS image).

(3) The spatial details are extracted from the PAN image by guided filter with the help of MS image as the guidance image.

(4) Calculate the injection gain corresponding to each band. The final fused image is obtained by adding the detail with the injection gain to each MS band.

\subsection{Meng's method [11]}

In this method,the authors proposed a novel pansharpening method with guided filter based on three-layer decomposition. The PAN image is decomposed into three layers,i.e., the edge layer, the detail layer, and the low frequency layer, by considering the edge-preserving characteristics of the guided filter. The edge layer and detail layer are then injected into MS image by a proportional injection model.

Meng's pansharpening method can be described as follows:

(1) The pixel values of the original MS and PAN images are normalized to [0, 1]. Then, the histogram matching of the PAN image to the intensity component is performed, and the intensity component is a linear combination of the bicubic resampled MS image. The linear combination coefficients are calculated by original MS image and the downsampled PAN image with least square regression.

(2) The histogram-matched PAN image is decomposed into three layers, a strong edge layer, a detail layer, and a low frequency layer.The guided filter is applied to decompose the histogram-matched PAN image into a base layer and a detail layer. The strong edge layer is separated from the base layer.

(3) The edge layer and the detail layer are injected into each MS band by a proportional injection model to obtain the fused image.

\section{Experimental results and analyses}

For analysis and comparison of the above and existing methods, fourbands (red, blue, green, and near infrared) multispectral IKONOS and QuickBird data are used. The spatial resolution for IKONOS satellite 
images are $1 \mathrm{~m}$ and $4 \mathrm{~m}$ for PAN and MS images respectively. The spatial resolution for QuickBird satellite images are $0.6 \mathrm{~m}$ and $2.4 \mathrm{~m}$ for PAN and MS images respectively. Relevant parameters are consistent with the original paper.

All the methods are evaluated from both qualitative and quantitative aspects. For qualitative evaluation, a true color display of the fused image is visually inspected to observe whether the objects are clear and the colors are natural and similar to the original MS image. Quantitative analysis is performed using correlation coefficient(CC), spatial CC(SCC), relative dimensionless global error in synthesis(ERGAS), spectral angle mapper(SAM), universal image quality index(UIQI), root mean squared error(RMSE), and Q4index[13 - 15].

\subsection{Experiment on IKONOS dataand analysis}

This experiment was carried out on IKONOS data. The results are shown in Figure 1. Figures 1(c) - (i) show the fused results of GS, GIHS, IAIHS, MGF, GFIHS, GFCS-B, GFCS-M, and Meng's methodrespectively.From the subjective perspective, the fusion methods based on guided filteraremuch better than the otherfusion methods without guided filter. The spectral distortion of the GS and GIHS methods(Figure 1(c)and 1(d)) is clearly visible as colors do not match the original MS image.The color of vegetation areas changes from dark green to light green.IAIHS fused result(Figure 1(e)) showsbetter quality thanGS and GIHS, but some pixelsappeared around the high reflectance edges such as the white roof of the building reduce huge spectral distortion.MGF method (Figure 1(f)) gives clear spatialdetail owing to the detailsextraction by multistage guided filter, however, the fused image suffers from over injection of the high frequency information from the PAN image. GFIHS method(Figure $1(\mathrm{~g})$ )maintains the spectral quality of the MS image without improving the spatial quality, and the result is blurred compared with other methods. The two methods GFCS-B and GFCS-M(Figure1(h) and 1(i)) from the subjective point of view are overall better than other methods. The disadvantage is that the injection of spatial information is not slightly enough. The result image of Meng's method (Figure 1(j))is different from the original image of the overall tone.

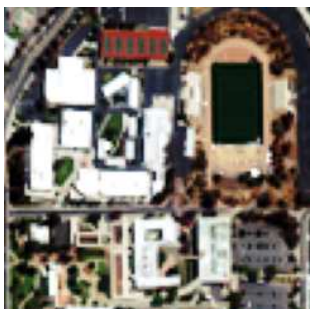

(a)

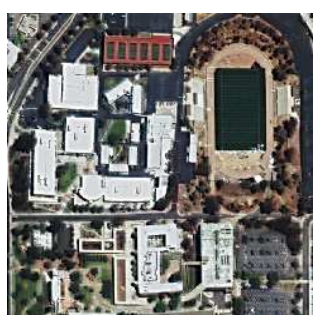

(f)

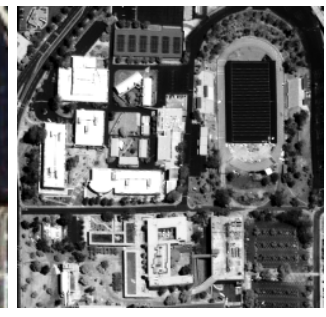

(b)

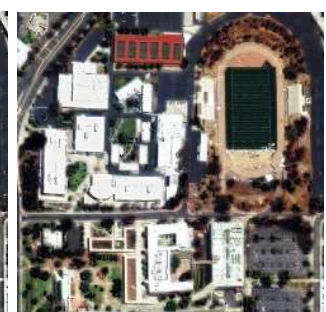

(g)

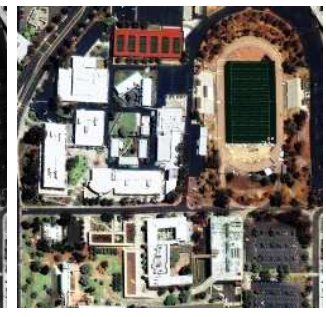

(c)

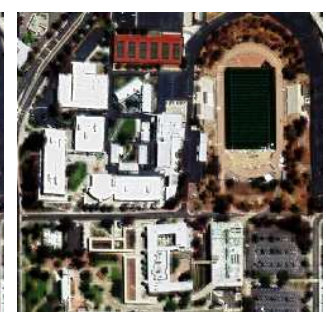

(h)

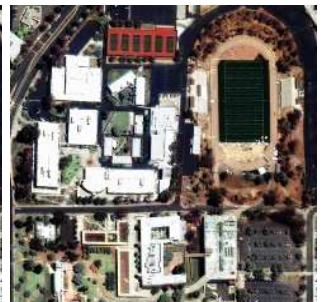

(d)

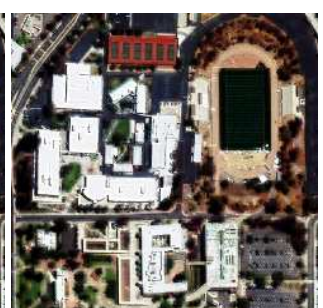

(i)

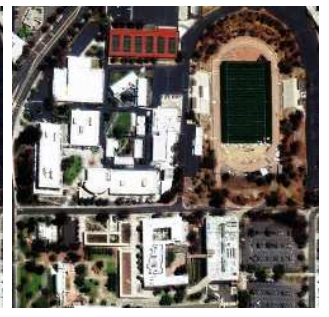

(e)

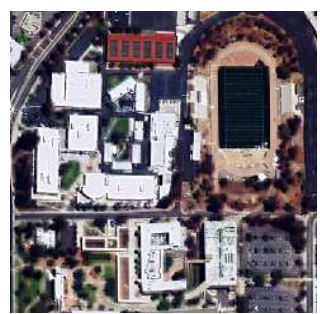

(j)

Figure 1. Fusion results of IKONOS satellite images.(a) MS image; (b)PAN image; (c)GS fusion result; (d)GIHS fusion result; (e)IAIHS fusion result; (f)MGF fusion result; (g)GFIHS fusion result; (h)GFCS-B fusion result; (i)GFCS-M fusion result; (j) Meng's fusion result.

Table 1. Quality indices of the fused images forIKONOS satellite

\begin{tabular}{lcccccccc}
\hline \multicolumn{1}{c}{ Index } & GS & GIHS & IAIHS & MGF & GFIHS & GFCS-B & GFCS-M & Meng's \\
\hline $\mathrm{CC}_{\mathrm{AVG}}(1)$ & 0.904 & 0.852 & 0.918 & 0.877 & 0.948 & 0.937 & $\mathbf{0 . 9 4 9}$ & 0.947 \\
$\mathrm{SCC}_{\mathrm{AVG}}(1)$ & 0.989 & $\mathbf{0 . 9 9 5}$ & 0.934 & 0.962 & 0.952 & 0.935 & 0.935 & 0.930 \\
RMSE $_{\mathrm{AVG}}(0)$ & 34.091 & 41.293 & 33.071 & 38.728 & $\mathbf{2 4 . 9 8 9}$ & 39.684 & 26.594 & 26.026 \\
UIQI $_{\mathrm{AVG}}(1)$ & 0.510 & 0.426 & 0.537 & 0.478 & 0.600 & 0.607 & $\mathbf{0 . 6 4 8}$ & 0.624 \\
ERGAS $(0)$ & 7.756 & 9.620 & 7.453 & 8.767 & $\mathbf{5 . 6 5 4}$ & 6.712 & 5.988 & 5.862 \\
$\mathrm{SAM}(0)$ & 6.696 & 16.809 & 3.756 & 9.851 & 4.588 & 3.279 & $\mathbf{2 . 2 2 4}$ & 7.153 \\
$\mathrm{Q} 4(1)$ & 0.825 & 0.763 & 0.826 & 0.790 & 0.881 & 0.862 & $\mathbf{0 . 8 8 2}$ & 0.880 \\
\hline
\end{tabular}


Table 1 reports the quantitative results in terms of seven quality indices for each method. From Table 1we can see that the methods based on guided filter yield the best pansharpening performance in terms of most of the image quality indexes. The indices ofCC $\mathrm{AVG}_{\mathrm{A}}, \mathrm{UIQI}_{\mathrm{AVG}}$, SAM, and Q4 measures based on GFCS-M method are better than other methods. However, RMSE $\mathrm{AVG}_{\mathrm{G}}$ and ERGAS are the highest using GFIHS. Generally speaking, GFCS-M method produces the impressive quality pansharpened result.

\subsection{Experiment on QuickBird data and analysis}

This experiment was carried out on the QuickBird data. The results are shown in Figure 2. Figures 2(c) - (j) show the fused results of GS,GIHS, IAIHS, MGF, GFIHS, GFCS-B, GFCS-M, andMeng's method respectively. Here, the GS and GIHS methods(Figure 2(c) and 2(d)) lack in preserving the spectral contents when compared to other methods. Individual pixel point distortion phenomenon of IAIHS method(Figure 2(e)) is obvious, and the edge of the high reflection area is more prone to distortion points, for instance, the white cars next to the road disappear.MGF method (Figure 2(f)) is the best space for details information, however its color intensity is very weak. GFIHS method(Figure $2(\mathrm{~g})$ ) owns higher spectral quality compared to MGF and GFCS-B methods, but the detail of the injection is not enough. The image's edge is blurry. Spectral distortion of GFCS-Bmethod(Figure 2(h))is serious. The overall image's color become deep,and many of the details are lost. Spectral information of GFCS-M method(Figure 2(i))is close to the original MS image,but spatial detail information is worse than GFCS-B from subjective observation. Above these implemented methods, Meng's method(Figure 2(j)) gets the best result. The method not only obtains a good spatial effect, but also has a higher spectral fidelity than other methods.

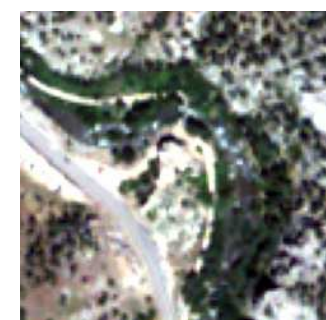

(a)

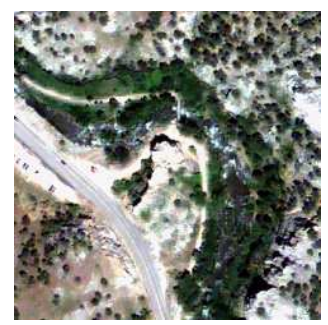

(f)

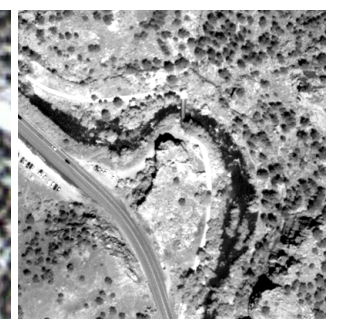

(b)

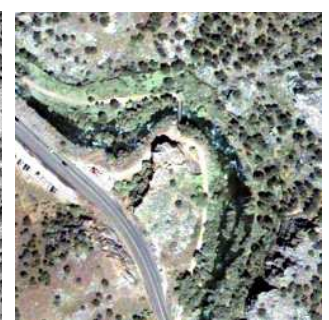

(c)

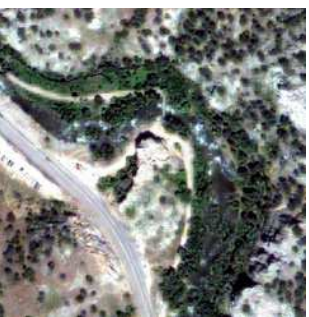

(g)

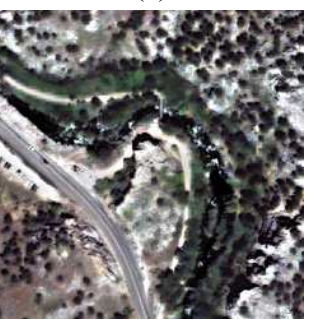

(h)

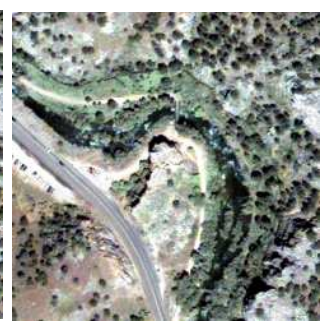

(d)

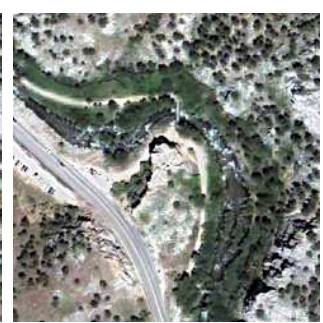

(e)

Figure 2. Fusion results of QuickBird satellite images.(a)MS image; (b)PAN image; (c)GS fusion result; (d)GIHS fusion result; (e)IAIHS fusion result; (f)MGF fusion result; (g)GFIHS fusion result; (h)GFCS-B fusion result; (i)GFCS-M fusion result; (j) Meng's fusionresult.

Table 2. Quality indices of the fused images forQuickBird satellite

\begin{tabular}{lccccccccc}
\hline \multicolumn{1}{c}{ Index } & GS & GIHS & IAIHS & MGF & GFIHS & GFCS-B & GFCS-M & Meng's \\
\hline $\mathrm{CC}_{\mathrm{AVG}}(1)$ & 0.704 & 0.794 & 0.927 & 0.887 & 0.954 & 0.871 & 0.933 & $\mathbf{0 . 9 7 7}$ \\
$\mathrm{SCC}_{\mathrm{AVG}}(1)$ & $\mathbf{0 . 9 8 3}$ & 0.978 & 0.748 & 0.921 & 0.891 & 0.844 & 0.839 & 0.826 \\
RMSE $_{\mathrm{AVG}}(0)$ & 54.426 & 44.173 & 26.622 & 35.828 & 21.94 & 37.895 & 26.419 & $\mathbf{1 6 . 6 7 0}$ \\
UIQI $_{\mathrm{AVG}}(1)$ & 0.361 & 0.442 & 0.576 & 0.495 & 0.615 & 0.573 & 0.629 & $\mathbf{0 . 7 5 3}$ \\
ERGAS $(0)_{\mathrm{SAM}(0)}$ & 9.076 & 12.167 & 5.641 & 8.085 & 4.721 & 7.822 & 5.440 & $\mathbf{3 . 4 2 4}$ \\
$\mathrm{Q} 4(1)$ & 9.447 & 17.780 & 4.538 & 9.095 & 5.555 & 7.109 & $\mathbf{3 . 1 9 5}$ & 4.041 \\
\hline
\end{tabular}

Table 2 reports the quantitative results in terms of seven quality indices for each method. From Table 2 we can see the methods based on guided filter have relatively slight better fusion performance than other methods, especially Meng's method. For QuickBird data, this method owns the best quality indices tested above exceptSCC $\mathrm{CVG}_{\mathrm{AV}}$ andSAM. 


\section{Conclusion}

Fusion of multispectral and panchromatic images is one of the most important technologies for processing remote sensing information. This paper briefly reviews several pansharpening methods based on guided filter, such as MGF, GFIHS, GFCS-B/GFCS-M, andMeng's method, as well as GS, GIHS, and IAIHS method.Experimental results from IKONOS and QuickBird satellite data have shown that guided filter based pansharpening methods perform better in retaining the spectral information of the original MS image and improving their spatial qualities.Among these methods, GFCS-M method is the most appropriatefor IKONOS data, moreover,Meng's method is the most appropriatefor QuickBird data.In summary, it confirms that guided filter is more suitable for remote sensing image fusion.Although these methods have relatively good performance, it is still necessary forresearchers to investigate further.The future work may focus on improving the guided filter based fusion model to improve the fused quality.

\section{Acknowledgement}

This work is sponsored by the Seed Foundation of Innovation and Creation for Graduate Students in Northwestern Polytechnical University.

\section{References}

[1] Camps-Valls G., Benediktsson J.A., Bruzzone L., et al. Introduction to the issue on advances in remote sensing image processing. IEEE Journal of Selected Topics in Signal Processing. 2011: $365-369$.

[2] Kumar S.S. and Muttan S. PCA based image fusion. Proceedings of SPIE. 2006, 6233 - 62331T.

[3] Laben C.A. and Brower B.V. Process for enhancing the spatial resolution of multispectral imagery using pan-sharpening.US Patent 6,011,875, 2000 .

[4] Wu J. Remote sensing image fusion based on average gradient of wavelet transform. IEEE Proceedings of the International Conference on Mechatronics and Automation. 2005: 1817 - 1821.

[5] Tu T.M., Huang P.S., Hung C.L., et al.A fast Intensity-Hue-Saturation fusion technique with spectral adjustment for IKONOS imagery. IEEE Geoscience and Remote Sensing Letters. 2004: 309 - 312.

[6] Legung Y., Liu J.M. and Zhang J.S.An improved adaptive Intensity-Hue-Saturation method for the fusion of remote sensing images. IEEE Geoscience and Remote Sensing Letters. 2014: 985 - 989.

[7] He K.M., Sun J., and Tang X.O. Guided image filtering. IEEE Transactions on Pattern Analysis and Machine Intelligence. 2013: 1397 - 1409.

[8] Upla K.P., Joshi S., Joshi M.V., et al. Multiresolution image fusion using edge-preserving filters. Journal of Applied Remote Sensing. 2015: 1 - 26.

[9] Jameel A., Riaz M.M., and Ghafoor A. Guided filter and IHS based Pan-Sharpening. IEEE Sensors Journal. $2016: 192$ 194.

[10] Liu J.M. and Liang S.L. Pan-sharpening using a guided filter. International Journal of Remote Sensing. $2016: 1777$ 1800.

[11] Meng X.C., Li J., Shen H.F., et al. Pansharpening with a guided filter based on three-layer decomposition. Sensors. 2016: $1-15$.

[12] Li S.T., Kang X.D., and Hu J.W. Image fusion with guided filtering. IEEE Transactions on Image Processing. 2013: $2864-2875$

[13] Kalpoma K.A., Kawano K., and Kudoh J. IKONOS image fusion process using steepest descent method with bi-linear interpolation. International Journal of Remote Sensing. 2013: 505 - 518.

[14] Yuhas R.H., Goetz A.F., and Boardman J.W. Discrimination among semi-arid landscape endmembers using the spectral angle mapper (SAM) algorithm. Summaries 3rd Annual JPL Airborne Geoscience Workshop. 1992: 147 - 149.

[15] Alparone L., Baronti S., Garzelli A., et al. A global quality measurement of pan-sharpened multispectral imagery. IEEE Geoscience and Remote Sensing Letters. 2004: 313- 317. 